\title{
La réforme des juridictions du travail
}

\section{Melda Sur}

\section{(2) OpenEdition}

\section{Journals}

Édition électronique

URL : https://journals.openedition.org/rdctss/2065

DOI : $10.4000 /$ rdctss.2065

ISSN : 2262-9815

\section{Éditeur}

Centre de droit comparé du travail et de la sécurité sociale

\section{Édition imprimée}

Date de publication : 1 avril 2018

Pagination : 192-195

ISSN : 2117-4350

\section{Référence électronique}

Melda Sur, «La réforme des juridictions du travail », Revue de droit comparé du travail et de la sécurite sociale [En ligne], 1 | 2018, mis en ligne le 01 novembre 2021, consulté le 12 novembre 2021. URL http://journals.openedition.org/rdctss/2065 ; DOI : https://doi.org/10.4000/rdctss.2065

\section{(c) (i) (9)}

Revue de droit comparé du travail et de la sécurité sociale est mise à disposition selon les termes de la Licence Creative Commons Attribution - Pas d'Utilisation Commerciale - Pas de Modification 4.0 International. 


\section{MELDA SUR}

UNIVERSITÉ DOKUZ-EYLÜL

\section{LA RÉFORME DES JURIDICTIONS DU TRAVAIL}

Adoptée à l'issue d'une longue période de gestation, la loi n 7036 du 12 octobre 2017 sur les tribunaux du travail (İs Mahkemeleri Kanunu) ${ }^{1}$ vient remplacer l'ancienne loi de $1950^{2}$. Le but principal de la réforme est de remédier à la surcharge de travail des juridictions sociales et en particulier des chambres sociales de la Cour de Cassation. Par cette réforme d'envergure, le législateur a essayé de traiter le problème sur plusieurs fronts.

\section{I - VUE D’ENSEMBLE}

L'exposé des motifs est éloquent: la durée moyenne des procès du travail en 2016, était de 434 jours, et parmi les 3525000 litiges en droit privé, $15 \%$ seraient des litiges du travail ${ }^{3}$. De surcroît, parmi les 780000 dossiers civils que la Cour de cassation a dû examiner, $30 \%$ concernaient les litiges du travail. Ces développements se trouvent liés à de nombreux facteurs, comme la croissance démographique, l'évolution des modalités d'exécution du travail, les progrès extraordinaires de la technologie, l'élargissement du champ de la sécurité sociale, la diversité et la multiplication des litiges du travail. La loi $n^{\circ} 7036$ apporte tout d'abord une modification nécessaire en accordant une compétence plus étendue aux tribunaux du travail, désormais compétents pour tout litige reposant sur la relation de travail (art. 5), quelle que soit la loi applicable au salarié. Ainsi, notamment le grand nombre de salariés travaillant sous contrat de travail soumis au Code des obligations (art. 393-469), comme les employés de maison, les ouvriers agricoles d'exploitations de moins de 50 salariés, les salariés (jusqu'à trois personnes) des petits commerçants et artisans, les pilotes et personnel de cabine dans l'aviation entreront désormais dans le champ de compétence des tribunaux du travail.

À côté de cette nouvelle compétence étendue, des modifications plus contestées ${ }^{4}$ ont été introduites dans le but de remédier à la surcharge de travail des juridictions:

- L'art. 21 du Code du travail régissant la protection contre le licenciement est amendé: les jugements auparavant «déclaratoires» se bornaient à constater la nullité du licenciement sans calculer les sommes dues si l'employeur refusait de réintégrer le salarié. Or, dans le secteur privé, l'employeur refuse souvent de réintégrer le salarié et préfère payer l'indemnité légale (quatre à huit mois de salaire et jusqu'à quatre mois de salaires pour la période avant la réintégration). Le salarié devait par la suite intenter un autre procès pour accéder à ses droits. Ainsi, désormais, le juge qui statue sur la nullité du licenciement devra également calculer le montant des indemnités et des créances

1 Journal Officiel du 25 octobre 2017, $\mathrm{n}^{\circ} 30221$.

2 Loi n 5521, du 0.1.1950, Journal Officiel du 4 février 1950, n 7424.

3 Les chiffres sont donnés par la Direction Générale du registre de justice et des statistiques (Adli Sicil ve Istatistik Genel Müdürlüğü).

4 Pour une évaluation d'ensemble voir notamment, A. Güzel, «İş Mahkemeleri Kanun Tasarısı Taslağı Hakkında Aykırı Düşünceler », Çalışma ve Toplum, 2016/3, nº 50, p. 1131-1146. 
dues $^{5}$. Toutefois, les dispositifs du jugement sur ces indemnités auront nécessairement un caractère conditionnel, puisque subordonnés aux cas de "refus de réintégrer le salarié », ce qui sera de nature à créer des difficultés pour leur exécution à ce stade ${ }^{6}$.

- Les délais de prescription, de dix ans selon la règle générale du Code des obligations ont été écourtés à cinq ans pour les créances les plus couramment réclamées lors de la rupture du contrat de travail: les congés-payés annuels, l'indemnité de licenciement (kıdem tazminatı), l'indemnité de préavis (ihbar tazminatı), l'indemnité de rupture abusive (kötüniyet tazminatı) et l'indemnité de discrimination (ayrımcılık tazminatı).

- La Loi modifie sensiblement la signification et la portée de la médiation régie par la loi sur la médiation dans les conflits juridiques ${ }^{7}$, pour la rapprocher un peu de la médiation dans les conflits collectifs de travail (art. 50 de la loi sur les syndicats et les conventions collectives de travail). Ainsi le médiateur peut désormais " proposer une solution » si les parties ne parviennent pas à un accord (art.17 modifiant les articles 2 et 15 de la loi sur la médiation dans les litiges juridiques).

Toutefois, les changements les plus significatifs et les plus discutés sont, la phase de médiation devenue obligatoire pour une grande partie des litiges du travail (art. 3) et la suppression du pourvoi en cassation pour certains jugements (art. 8).

\section{II - LA MÉDIATION CONÇUE COMME PHASE PRÉALABLE OBLIGATOIRE}

\section{A - PRINCIPALES DISPOSITIONS}

Dans le but de remédier à la charge excessive des juridictions du travail, le recours à la médiation devient obligatoire avant l'ouverture de tout procès sur des litiges du travail portant sur des créances et indemnités légales, contractuelles ou fondées sur une convention collective de travail, ainsi que la réintégration de travailleurs licenciés. Exception est faite en matière d'accidents ou de maladies du travail (par. 3 de l'art. 3), sujets intéressant de plus près l'ordre public. Pour les actions susmentionnées, les parties qui ont omis de passer par cette phase se verront opposer une fin de non recevoir lors de l'ouverture du procès. La loi organise concrètement la médiation, comme la Loi $n^{\circ} 6325$ sur la médiation dans les litiges, également applicable. Ainsi sont définis les qualifications requises du médiateur (diplôme de droit, formation spéciale, absence de certaines condamnations, impartialité et indépendance), les bureaux de médiation compétents, le choix du médiateur - laissé aux parties sur une liste rendue publique, ou effectué par le bureau en cas d'absence d'accord sur la liste officielle. Une aide judiciaire sera accordée en cas de besoin selon les dispositions du Code de procédure civile.

La durée de la médiation est de trois semaines, que le médiateur peut prolonger jusqu'à une semaine si nécessaire. Notons que lorsque l'échec de la médiation est dû à l'absence - non excusable - de l'une des parties à la première réunion, celle-ci sera plus tard tenue du remboursement de la totalité des frais judiciaires, même si elle obtient gain de cause, et ne pourra pas non plus réclamer les frais d'avocat (par. 12 de l'art. 3). Si les deux parties s'absentent à la première réunion, les frais seront à charge égale, et de manière générale, la rémunération du médiateur est partagée en parts égales par les parties. Par contre, s'il y a

5 Voir, Ü. Narmanlıoğlu, "İş Kanunu'nun İşçiye Güvence Sağlayan Feshin Geçersizliği Davasının Yeni Çerçevesi (İş Mahkemeleri Kanunu'nun Feshin Geçersizliği Davasında Yaptığı Değişiklikler)», Sicil, 2017, $\mathrm{n}^{\circ} 38$, p. 9-21.

6 Voir, M. Astarlı, «7036 Sayılı İş Mahkemeleri Kanunu'nun 4857 Sayılı İş Kanunu'nun İş Güvencesi Hükümlerinde Öngördüğü Değişikliklerin Değerlendirilmesi », Sicil, 2017, nº 38, p. 40-53.

7 Loi n 6325 du 7.6.2012 (Hukuk Uyuşmazlıklarında Arabuluculuk Kanunu). 
échec au bout de deux heures, la rémunération sera effectuée par le Trésor public. Dans les cas où la médiation aboutit à un accord, celui-ci revêtira un caractère exécutoire après avoir été visé par le juge de paix. Les signatures du médiateur et des deux parties ainsi que de leur avocat donnent à cet accord le caractère d'un « jugement exécutoire »(art.18, par. 2, 3 et 4 de la loi sur la médiation dans les litiges juridiques).

\section{B - CRITIQues et RÉFÉRENCES}

L'obligation du recours à la médiation a été très critiquée dans la doctrine. L'argument de base des détracteurs se fonde sur le caractère impératif de la plupart des dispositions tendant à la protection du travailleur et qui caractérisent la législation sociale. Or, le travailleur sera souvent amené à accepter un compromis désavantageux et à renoncer à certains de ses droits. L'inégalité de fait des parties entacherait l'accord auquel la médiation va aboutir, et justice ne serait pas faite ${ }^{8}$. Si l'on se réfère à la Recommandation Rec (2002) 10 du Comité des Ministres du Conseil de l'Europe, de tels accords semblent admis mais avec une certaine circonspection: «16. Un document écrit devrait d'ordinaire être rédigé à la fin de chaque processus de médiation pour définir l'objet, l'étendue et l'issue de l'accord. Les parties devraient pouvoir bénéficier d'un délai de réflexion limité et agréé entre les parties entre le moment où il est rédigé et la date de sa signature ». "17. Les médiateurs devraient informer les parties de l'effet des accords issus de la médiation et des démarches à suivre si l'une ou les deux parties souhaitent exécuter leurs accords. De tels accords ne devraient pas aller à l'encontre de l'ordre public ».

Reste à savoir si «l'ordre public social » peut ainsi être mis de côté. Néanmoins, le texte semble laisser aux États une certaine latitude: «11. Les États devraient décider si, et dans quelle mesure les clauses relatives à la médiation peuvent restreindre le droit des parties d'agir en justice. " Ainsi, dans un passé récent, un recours devant la Cour constitutionnelle concernant la loi sur la médiation sur les litiges juridiques avait été rejeté, la Cour ayant fondé sa décision principalement sur le caractère consensuel de l'accord auquel aboutissent les parties qui ne sont point tenues de parvenir à un quelconque accord, et peuvent ensuite ester en justice ${ }^{9}$. Toutefois, la Cour a statué à l'époque alors que le recours à la médiation n'était que facultatif. Quelle serait la solution face à un recours à la médiation rendu obligatoire dans les litiges sociaux les plus importants ce recours étant une condition de recevabilité de l'action en justice? La réponse est incertaine ${ }^{10}$. Malgré les transformations sociétales importantes, l'inégalité des parties demeure.

8 Voir not. les actes de la conférence du 14 mai tenue à Istanbul, organisée par l'Association du Droit du travail et de la sécurité Sociale (Iss Hukuku ve Sosyal Güvenlik Hukuku Derneği 40. Yıl Uluslararası Toplantısı, iş Mahkemeleri Kanunu Tasarısı Taslağının Değerlendirilmesi, Ed. K. Doğan Yenisey): M. Namlı, İş Mahkemeleri Kanunu Tasarısı Taslağı Ille Getirilen Zorunlu Arabuluculuk Kurumunun Medenî Usul Hukuku Bakımından Değerlendirilmesi, p. 151-165; K. Doğan Yenisey, İş Yargısında Zorunlu Arabuluculuk, p. $167-$ 193; G. Alpagut, İş Mahkemeleri Kanunu Tasarı Taslağı ile Bazı Kanunlarda Değişiklik Yapılmasına Dair Kanun Tasarısı Taslağının Değerlendirilmesi, p. 195-217.

9 Cour constitutionnelle, arrêt du 10 Juillet 2013, 2012/94-2013/89, Journal Officiel du 25 janvier 2014, $\mathrm{n}^{\circ} 28893$.

10 Dès le 26 octobre 2017, le Conseil National des Barreaux (Türkiye Barolar Birliği) a publié une déclaration (Duyuru 2017/61) selon laquelle certaines dispositions de la Loi ne seraient point conformes à la Constitution. Parmi celles-ci, l'obligation de recours à la médiation avant d'ester en justice, le caractère définitif et insusceptible de recours en justice des points où il y a eu accord, les frais de médiation considérés comme frais judiciaires et la possibilité de représentation de l'employeur par son salarié lors de la médiation. Les tribunaux peuvent eux-mêmes référer ces dispositions à la Cour Constitutionnelle (art.40 de la Loi n 6216 sur l'instauration et le fonctionnement de la Cour Constitutionnelle). 


\section{III - LA SUPPRESSION DU POURVOI EN CASSATION CONTRE CERTAINES DÉCISIONS}

Le législateur complète la médiation par une autre mesure concernant les voies de recours judiciaires. En effet, après l'entrée en fonction des Cours d'appel (Bölge Adliye Mahkemeleri) en Juillet 2016, le problème de la durée des procès se pose avec plus d'acuité et il s'agissait donc d'apporter une correction à la multiplication des voies de recours. Le législateur a ainsi rendu définitifs certains arrêts des Cours d'appel, indépendamment de la valeur du litige. Les procès aux fins de réintégration de salariés licenciés (art. 21 du Code du travail et art. 24 de la loi sur les syndicats et les conventions collectives de travail) forment une part importante de ces litiges. Dans le domaine de la négociation collective, la détermination de l'unité de "négociation d'entreprise » (işletme toplu iş sözleşmesi, art. 34 de la loi sur les syndicats et les conventions collectives de travail), les sanctions disciplinaires de l'employeur, l'interprétation de la convention collective (art. 59/1) et le caractère licite ou illicite de la grève ou du lock-out (art. 71/1) font également partie des sujets de litiges fermés au contrôle de la Cour de cassation. Se pose alors la question de l'indispensable rôle de la Cour de cassation dans l'interprétation des normes, le développement du droit et son adaptation aux besoins, et l'élaboration de principes. La jurisprudence de la Cour de cassation a largement servi de source d'inspiration au législateur, en apportant des solutions nouvelles et adaptées aux réalités. Enfin, la Cour de cassation a une fonction essentielle qui est d'assurer l'unité de la jurisprudence. C'est pourquoi, la réforme suscite des inquiétudes justifiées ${ }^{11}$.

\section{Conclusion}

L'élargissement du champ de compétence des juridictions du travail à l'ensemble des litiges fondés sur la relation de travail est une réforme bienvenue, dont les incidences seront de faible ampleur sur la charge des tribunaux du travail. Par contre, en donnant un caractère définitif à une importante partie des arrêts des Cours d'appel (notamment dans l'application de la protection contre le licenciement), tout un pan des litiges sociaux va se trouver soustrait du contrôle de la Cour de cassation dont l'un des rôles fondamentaux est d'assurer l'unité de la jurisprudence. Quant à la médiation, le Comité des Ministres du Conseil de l'Europe, en sa Recommandation $n^{\circ} \mathrm{R}(86) 12$ du 16 septembre 1986 relative à certaines mesures visant à prévenir et réduire la surcharge de travail des tribunaux avait bien invité les gouvernements à " encourager, dans les cas appropriés, le règlement amiable des différends, soit en dehors de l'ordre judiciaire, soit avant ou pendant la procédure judiciaire ». À cet effet, parmi les mesures envisageables, les États pouvaient " prévoir, avec des avantages appropriés, des procédures de conciliation qui, préalablement à la procédure judiciaire ou en marge de celle-ci, auraient pour but de régler le litige ». Or ici le risque est grand, avec l'introduction de la phase de médiation obligatoire, de voir battue en brèche une législation du travail qui vise à protéger le salarié qui peut être amené à renoncer à une partie de ses droits, ces droits-mêmes que le législateur a essayé de garantir par une législation sociale protectrice.

11 Dans ce sens not: Güzel, Çalışma ve Toplum, 2016/3, p. 1137-1140. 